\title{
Quaderni
}

QUADERNI Communication, technologies, pouvoir

\section{Mise en cause de l'autorité médicale et légitimation du discours d'expérience sur les forums de discussion en ligne}

\section{Aurélia Lamy}

\section{OpenEdition}

Journals

Édition électronique

URL : http://journals.openedition.org/quaderni/1074

DOI : 10.4000/quaderni. 1074

ISSN : 2105-2956

Éditeur

Les éditions de la Maison des sciences de l'Homme

Édition imprimée

Date de publication : 5 mai 2017

Pagination : 43-52

Référence électronique

Aurélia Lamy, "Mise en cause de l'autorité médicale et légitimation du discours d'expérience sur les forums de discussion en ligne », Quaderni [En ligne], 93 | Printemps 2017, mis en ligne le 05 mai 2019, consulté le 02 janvier 2020. URL : http://journals.openedition.org/quaderni/1074 ; DOI : 10.4000/ quaderni. 1074 


\section{$D$ ossier}

\section{Mise en cause} de l'autorité médicale et légitimation du discours

d'expérience sur les forums de discussion en ligne

Aurélia Lamy

Maître de conférences GERIICO Université de Lille 1
Dans le domaine de la santé, l'autorité médicale est incarnée par le médecin qui est, par son statut professionnel et la légitimité sociale dont il bénéficie, le plus à même d'établir un diagnostic et assurer le suivi médical d'un patient. L'autorité médicale semble être de fait dans une société où on considère que «l'autorités'acquiert, au fur et à mesure où un champ de connaissances précises se dessine, et conduit à la prise de conscience que le médecin "sait" là où les autres ne "savent" pas» (Ménard, 2008 : 247). Le médecin bénéficie en ce sens d'une sorte de "domination légale rationnelle» (Weber, 1971) fondée sur son statut, son expérience, ses compétences et légitimée par l'institution qu'il représente. Il apparaît en cela comme une "figure d'autorité » (Herfray, 2005) face au patient. Dans la relation médecinpatient, cette notion d'autorité implique une relation de pouvoir, instaurée par une inégalité de savoir entre le médecin et le patient ; relation pérennisée par la légitimité conférée aux discours médicaux par l'institution qui les contraint mais également par les discours sociaux qui tendent à les valoriser. Pourtant, de nombreux facteurs peuvent conduire à la remettre en cause : la religion (Fainzang, 2002), le sentiment d'autoefficacité (Moriarty et al., 2008), la subjectivité des perceptions (Ménard, 2008) souvent alliée à la connaissance préalable de la maladie par le patient et les pratiques informationnelles.

L'apparition des nouvelles technologies a conforté cette mise à distance/relativisation de l'information médicale donnée par le médecin. Les patients sont aujourd'hui plus (mieux ?) informés, via internet et notamment les forums de discussion qui favorisent un échange asynchrone, des « conversations discontinues » (Marcoccia, 
2003) sur des thématiques définies et cadrées où chacun peut être à son tour éditeur, lecteur, " modérateur », et où «la mise en scène de soi et l'interprétation de la conduite de l'autre prennent [...] des formes spécifiques, qui utilisent les ressources du contexte : l'écrit électronique, le multimédia, les manières de se comporter dans l'espace du forum, la maîtrise technique du medium, la familiarité avec les règles de conduite locales... » (Beaudouin, Velkovska, 1999 : 145). Si les forums sont des espaces privilégiés de circulation de $1^{\prime}$ " information de santé ${ }^{1}$ », ils induisent une "scénographie particulière" (Monte et Oger, 2015 : 6) qui contribue fortement à la (dé)construction de l'autorité et forment ainsi des espaces où peuvent être discutés les figures de l'autorité médicale.

Dans cet article nous avons choisi de travailler sur deux forums de discussion publiés sur le site Les impatientes :

$1 /$ « Dossier et jargon médical » - Blog Les impatientes - 312 messages entre le 29/06/2006 et le 16/08/2015

2/ « Changer la relation patient-médecin »Blog Les impatientes - 336 messages entre le 05/07/2006 et le 16/10/2015 .

Premier réseau de femmes atteintes du cancer du sein, Les impatientes a vocation à permettre aux patientes de réinvestir l'information médicale et est en cela destiné à celles qui veulent « être au cour de l'équipe soignante, active », à «celles qui ne veulent plus subir la médecine en étant simples patientes, [à] celles qui ont envie de prendre leur santé en main. ${ }^{4}$ » Les forums sélectionnés ont pour vocation d'expliciter, d'une part, les difficultés de compréhension du « jargon » médical qui apparaît parfois comme un frein à l'appréhension de la maladie par les patientes et, d'autre part, de mettre des mots sur le vécu du lien médecin-patient, sur cette relation de «domination » légitimée par l'institution mais parfois remise en question par les patientes. Ainsi, notre objectif est bien de mieux comprendre comment la figure d'autorité incarnée par le médecin - généraliste ou spécialiste - peut être remise en question par les patientes elles-mêmes dans des espaces de discussion extra-médicaux, mais également comment se construit, à travers les discours et les dispositifs d'énonciation, une autorité d'expérience au sein d'une communauté en ligne où l'information de santé vient, non pas suppléer, mais expliciter, éclairer et compléter l'information médicale. D'un point de vue méthodologique, ces deux forums donnent lieu à une analyse de discours permettant de mettre en évidence les termes associés à l'autorité médicale mais également les procédés discursifs de remise en cause de cette autorité et les postures de légitimité adoptées par les patientes-internautes dans un dispositif de libre expression ouvrant des possibilités mais également porteur de contraintes.

\section{Le forum de discussion : une alternative à la confrontation médicale}

Les intervenantes des forums sélectionnés sont des femmes touchées directement par un cancer du sein. La manière dont elles se définissent : " les impatientes », les « essentielles » et dont elles s'adressent les unes aux autres : «les filles», « les copines », « mes douces amies » montre à quel point les échanges perçus dans le cadre des forums permettent la création de liens interpersonnels forts. Ainsi, participer aux discussions 
c'est rejoindre une « grande famille » (Vivie) qui fait front, ensemble, contre la maladie. Ici, « les locuteurs sont particulièrement reconnus, au sens où ils sont censés détenir un savoir, une compétence, une position, une expérience, un savoir-faire qui autorisent leur parole » (Monte et Oger, $2015: 7$ ), les internautes bénéficient en cela d'une autorité de source reposant d'une part « sur des critères supposés rationnels » (touchées par la maladie, elles disposent d'un savoir d'expérience) et/ou « sur une "déférence" socialement construite » (Orrigi, 2004 ; Id.,2008 : 43-49, cité par Monte et Oger, $2015: 7$ ) particulièrement perceptible dans les échanges formalisés au sein du forum.

Pour favoriser ces interactions et mieux comprendre « qui parle » et « de quel point de vue », l'usage du forum implique la définition d'une « identité numérique » (Pinte, 2014). En effet, le processus d'interaction que permettent ces dispositifs, au-delà de l'identité virtuelle (Georges, 2010) affichée, fait émerger des identités situées, discursives et octroyées (Beaudouin et Velkovska, 1999) qui contribuent à la construction de l'identité collective en ligne et à l'adoption de postures d'autorité. Certaines internautes s'octroient par exemple un rôle de modérateur dans les discussions, non pas dans l'objectif de brimer les discours ou recadrer le débat mais pour permettre à toutes d'obtenir des réponses adaptées à leurs questions : «tu aurais plus de chances d'avoir des réponses ou peut-être même d'en trouver dans les messages existants $d u$ forum grossesse et cancer du sein »(Suzzzz***), l'internaute se positionne ainsi en utilisatrice avertie du forum et donc en ressource légitime lorsqu'il est question de son utilisation et de l'exploitation de son contenu.

Sur l'ensemble des 648 posts concernés par notre étude, 217 Impatientes s'expriment. L'analyse de ces posts montre que la présence des impatientes sur ce site peut être motivée différemment selon leur ancienneté et leur connaissance de la maladie :

- Certaines viennent sur le forum qu'elles considèrent comme une alternative à la consultation médicale ; cette première démarche d'information est moins engageante puisqu'elle n'induit pas de co-présence et garantit l'anonymat et la liberté de parole ;

- D'autres estiment que le temps d'attente avant le prochain rendez-vous médical est trop long et souhaitent recueillir des informations en amont de ce rendez-vous pour se rassurer ou partager leurs inquiétudes ;

- D'autres enfin, n'estiment pas la réponse médicale satisfaisante et viennent sur les forums pour obtenir des informations complémentaires, des explications, des éclairages quant aux incompréhensions auxquelles elles font face. Elles viennent parfois aussi compléter les informations médicales dont elles disposent avant ou après diagnostic.

Les forums sont donc pour la majorité d'entre elles une source d'informations supplémentaires sur leur maladie face à d'autres médias (internet et télévision en première ligne) où les informations disponibles sont souvent remises en cause. Cette confiance dans les sources recherchées est primordiale dans l'appréhension des informations. L'autorité dont il est ici question s'apparente à une forme d'autorité cognitive telle que définie par Wilson (1983), c'est-à-dire « une relation 
d'influence de pensée impliquant au minimum deux personnes, l'une accordant à l'autre sa confiance parce qu'elle maîtrise un domaine spécifique de compétences » (Broudoux, 2007). Les forums sont l'outil privilégié de mise en scène de cette forme d'autorité basée sur la confiance, la crédibilité, la réputation (perceptible dans les activités de l'internaute affichées sur le site) qui va bien au-delà du discours d'expert.

Ainsi, les forums étudiés ont d'une part un usage cathartique, d'autre part un rôle informatif. En ce sens, le dispositif choisi par les impatientes n'est pas anodin. En effet, les forums suscitent des interactions interpersonnelles visibles par toutes qui permettent à celles qui ne souhaitent pas intervenir de bénéficier des informations diffusées. À la lecture des forums, s'affirme une communauté de femmes concernées par la maladie, proposant une dynamique interpersonnelle forte marquée par le tutoiement de fait, des marques de gentillesse et d'intercompréhension, le partage d'émotions et d'informations de l'ordre de l'intime portées ici au regard de tous. Les forums deviennent alors le point de départ de la constitution d'un groupe autour duquel se développe une véritable expertise. En cela, internet peut devenir le support d'une activité qui concurrence frontalement l'autorité du monde médical.

\section{Légitimation ou remise en cause de la relation médecin-patient ?}

Il est difficile d'aborder la question de l'autorité médicale sans interroger la relation médecin-patient. En situation d'interaction, l'intercompréhension et la collaboration patientmédecin sont fondamentales et doivent conduire à une «négociation de sens » (Fabre et al., 2009) légitimant d'une part l'information médicale et l'autorité du médecin, d'autre part le choix du parcours de soin. Outre cette " information médicale " proférée dans un contexte légitime, officiel, institutionnel, le patient a aujourd'hui la possibilité de s'informer sur d'autres espaces, notamment en ligne. Ces nouvelles pratiques d'information en ligne peuvent inquiéter, en ce qu'elles touchent un domaine sacré d'un point de vue social, la santé. Et pourtant, les médecins eux-mêmes reconnaissent les bienfaits d'internet dans la relation médicale. Broom (2005 abc), montre en effet qu'Internet peut contribuer à faciliter le travail des professionnels de la santé en ce qu'il responsabilise les patients, renforce leur implication vis-à-vis du parcours de soin : « ainsi, Internet n'aurait pas tant contribué à la perte d'autorité des professionnels qu'à l'apparition de processus d'adaptation complexes et variés 》 (Akrich et Méadel, 2009). Par ailleurs, certaines études montrent que le processus de modération par les pairs sur les forums de discussion notamment garantit la véracité des informations (Esquivel et al., 2006) . $^{5}$ Ainsi, "le caractère collectifde l'activité sur Internet prémunit contre la désinformation ", "le niveau d'expertise des "profanes" apparaît donc élevé, puisque très peu d'erreurs circulent et qu'elles sont immédiatement repérées » (Akrich et Méadel, 2009). On constate cette dynamique dans les forums étudiés, en effet, les « impatientes » sont principalement sources d'informations : elles donnent des références, aident à la compréhension des symptômes de la maladie, sont parfois amenées à traduire des résultats d'analyse, voire à poser un diagnostic à partir de symptômes décrits. Ce savoir profane n'entre pas de fait en opposition avec les discours 
médicaux mais peut contribuer à la remise en question de l'autorité du discours médical.

Au fil des discussions, deux types d'autorité peuvent ainsi être remis en question : l'autorité médicale (du médecin, de l'institution) et l'autorité de l'information médicale. En premier lieu, les dysfonctionnements de l'institution conduisent à remettre en cause la relation médicale et la prise en considération du patient par le corps médical, le temps de prise en charge constitue notamment une critique récurrente : "j'ai perdu 3 mois avec le rhumato, le cancéro et la radiothérapeute » (jhmjh***). Ensuite, si la relation médecin-patient est considérée comme primordiale par les impatientes qui considèrent qu' « une partie de la guérison se fait par les rapports que l'on a avec le médecin et avec les échanges qui se font » (dandoune007), de manière frontale, se pose la question de la confiance, en effet, " l'action médicale suppose, comme sa condition de réalisation, l'installation d'une relation de confiance mutuelle entre le malade et son médecin, plus largement entre les malades et les autorités médicales » (Martin et Quiviger, 2007). Or, plusieurs éléments peuvent conduire le patient à douter des compétences de son médecin et ainsi remettre en cause son autorité. En premier lieu, l'attitude des médecins est pointée du doigt par les impatientes : le rapport de domination est parfois jugé trop présent dans la relation médecinpatient : « certains médecins se prennent pour Dieu» (Vivie), ce qui conduit certains d'entre eux à déshumaniser la relation tout en imposant leur autorité : « dès qu'un être vivant a un pouvoir, il l'exerce, même si pour cela, il doit se conduire comme le dernier des salauds » (Badoux). Cette attitude est jugée dangereuse pour la compréhen- sion et l'acceptation du parcours de soin par le patient : "Certains praticiens "règnent" par la terreur, ne concevant leur rapport aux patients qu'au travers d'une panique (je pèse mes mots) qui leur assure une sorte de toute puissance " (angemarie). Si ces réactions véhémentes ne sont pas majoritaires dans les discours étudiés, les patientes regrettent surtout le peu de considération accordée aux malades : «j'ai eu des relations très pénibles avec cette oncologue : très froide, très pressée, aucune relation humaine [...]J'étais un dossier parmi d'autres !!!! ( (dandoune007). Cette considération est pourtant essentielle notamment lors de l'annonce du diagnostic considéré comme un moment clé de la relation médecin-patient. On constate que ces réserves résultent finalement d'une confrontation entre les attentes des patientes engagées dans un parcours de soin qui espèrent «quelques conseils, des mots rassurants [...] autre chose que ces chiffres froids » (Caracol) et la réalité des pratiques médicales - des discours, des actes, des attitudes - qui nécessitent de la part des médecins une prise de distance et une objectivation de la maladie.

Si ces réserves sont explicitement formulées par des internautes au fil des discussions, la dynamique collective semble toutefois renforcer l'autorité et la légitimité du médecin. En effet certains médecins sont valorisés pour leurs pratiques et leur humanité vis-à-vis des patientes, cette valorisation est plus associée à la mise en avant de qualités personnelles qu'à des compétences professionnelles : «je suis suivie à l'institut Gustave Roussy [...] il ne commence jamais la visite sans m'avoir demandé des nouvelles de ma famille. Il me connaît par cour et m'appelle "ma petite corse". C'est un homme 
bien, droit, quelqu'un qui prend ses responsabilités » (Larla***). Par ailleurs, si les « impatientes » possèdent et revendiquent un savoir sur la maladie, le médecin reste le premier interlocuteur, le plus légitime à évaluer la gravité d'une situation ou à interpréter les symptômes : " tu devrais te le faire confirmer par ton généraliste » (Anne). Ainsi, malgré les critiques formulées quant à la prise en charge médicale, les interactions et la relation médecin-patient, les « impatientes » ont conscience de l'importance de l'information médicale et du rôle primordial du médecin dans le parcours de soin : «même si on est pas obligé de gober bêtement tout ce que nous disent les médecins, il faut bien leur faire un peu confiance (à ceux qui le méritent) si on veut se laisser soigner! 》 (Sissy). Ainsi, les « impatientes » ne revendiquent pas un savoir médical à proprement parlé, voire s'en détachent pour mieux affirmer l'importance du discours - et par conséquent de l'autorité - du médecin.

\section{Conclusion}

Aller sur ces forums de discussion permet aux patientes atteintes de cancer du sein d'être davantage investies dans leur trajectoire de soin. En partageant leur expérience, leur vécu de la maladie, elles contribuent à produire de l'information de santé complétant une information médicale parfois difficile à appréhender pour les malades, " on voit ici comment la circulation des connaissances et des expériences que permet Internet multiplie les lieux dans lesquels se constituent des expertises concurrentes, voire conflictuelles » (Akrich et Méadel, 2009). Ainsi, "le web dit 2.0 offre la possibilité à l'internaute des'affranchir des règles communément établies de production et de diffusion de l'information, développant alors des pratiques qualifiées par E. Broudoux [2003] d'autoritatives » (Alloing, 2014) qui s'affranchissement des institutions de références. Finalement, la question de la confiance semble être au cœur de la définition de l'autorité telle qu'elle est évoquée dans ces forums : l'autorité médicale est valorisée dans une relation de confiance entre le médecin et son patient, l'autorité du discours d'expérience n'est possible que si l'identité numérique de son auteur suscite la confiance de la communauté. Ici, plusieurs figures de patientes émergent des discussions, de la consultante à l'experte, plus ou moins investies, leur expérience de la maladie et de l'usage des forums leur donne une légitimité auprès du groupe et contribue à structurer une communauté de femmes en quête de sens.

Ainsi ces forums ont un usage cathartique tout autant qu'informationnel, en ce qu'ils permettent d'humaniser la maladie, de la désacraliser tout autant que de la comprendre. Les discours proférés ne se positionnent pas en opposition au discours médical, ils viennent le compléter, l'amender, l'expliciter. Si les critiques relatives aux discours médicaux, à l'attitude des médecins ou à la gestion des institutions, sont récurrentes, elles sont surtout exprimées sur le forum considéré comme une soupape de décompression où l'échange de parole libre, anonyme, autorise les malades à exprimer leur colère, leurs regrets et surtout leur peur, sans prendre le risque de dénaturer la relation que les impatientes entretiennent avec leur(s) médecin(s). Dans les forums étudiés, l'autorité médicale n'est jamais à proprement parlé remise en cause, «on assisterait ainsi plutôt à une transformation de l'autorité des médecins 
qu'à sa remise en cause, d'autant que s'ils utilisent Internet, les médecins peuvent maintenir la "compétence asymétrique" qui est au cour de leur identité professionnelle » (Akrich et Méadel, 2009). En protégeant certains discours médicaux et en mettant en valeur des figures notables dans le corps médical, les impatientes contribuent finalement à protéger l'autorité médicale en rendant légitime son exercice.

\section{$R \cdot \dot{E} \cdot F \cdot E \cdot R \cdot E \cdot N \cdot C \cdot E \cdot S$}

AKRICH M. , MÉADEL C. , «Internet, intrus ou médiateur dans la relations médecin-patient? », in Santé, science et solidarité, 8 (2), 2009, pp. 87-92.

ALLOING C. , « Vers une approche instrumentale de l'identité numérique : les attributs identitaires comme structuration de l'environnement informationnel ? », in Pinte J-P., Enseignement, préservation et diffusion des identités numériques, Hermès Lavoisier, 2014, pp. 39-68.

BEAUDOUIN V. , VELKOVSKA J. , « Constitution d'un espace de communication sur internet (forums, pages personnelles, courrier électronique...) », in Réseaux, vol. 17, n 97, 1999, pp. 121-177.

BROOM A. , "The eMale : Prostate cancer, masculinity and online support as a challenge to medical expertise », in Journal of Sociology, 2005a, 41, pp. 87-104.

BROOM A. , " Medical specialists' accounts of the impact of the Internet on the doctor/patient Relationship», in Health, 9(3), 2005b, pp. 319-338. BROOM A. , «Virtually He@lthy : The Impact of Internet Use on Disease Experience and the Doctor-Patient Relationship ", in Qual Health Res, 15 (3), 2005c, pp. 325-345.

BROUDOUX E. , « Autoritativité, support informatique, mémoire », in Journées hypertextes, mémoire, fiction, Montréal, Canada, novembre 2003.

BROUDOUX E. , Construction de l'autorité informationnelle sur le web, A Document (Re)turn : Contributions from a Research Field in Transition, 2007.

ESQUIVEL A., MERIC-BERNSTAM F. , BERNSTAM E. , «Accuracy and self correction 
of information received from an internet breast cancer list : content analysis », in BMJ, 332 (7547), 2006, pp. 939-942.

EYSENBACH G. , KOHLER C. , «How do consumers search for and appraise health information on the world wide web ? Qualitative study using focus groups, usability tests, and in-depth interviews », in BMJ, 324 (7337), 2002a, pp. 573-577. EYSENBACH G. , POWELL J. , KUSS O., et al., «Empirical Studies Assessing the Quality of Health Information for Consumers on the World Wide Web : A Systematic Review », in JAMA, 287 (20), 2002b, pp. 2691-2700.

FABRE C., CONDAMINES A., REBEYROLLE J., TANGUY L., VERGELY P. , « Analyse linguistique des disparités dans les échanges médecin-patient ", in Colloque de l'ADELF (Association des Épidémiologistes de Langue Française), 2009, Toulouse, France.

FAINZANG S. , «Les patients face à l'autorité médicale et à l'autorité religieuse », in Raymond Massé, Jean Benoist (dir.), Convocations thérapeutiques du sacré, Paris, Collection Médecines du monde, Les Éditions Karthala, 2002.

GEORGES F., Identités virtuelles. Les profils utilisateurs du web 2.0, Questions théoriques, 2010. HERFRAY C. , Les Figures d'autorité, Toulouse, Eres, 2005.

KIRSCHNING S. , Von KARDORFF E. , «The use of the Internet by women with breast cancer and men with prostate cancer-results of online research », in Journal of Public Health, 16 (2), 2008, pp. 133-143.

LE GOFF-PRONOST M. , FARAJALLAH M. , PENARD T. , SUIRE R., " Quoi de neuf docteur? Une étude économétrique sur la recherche en ligne d'informations médicales par les patients », in XXXI Jes Journées des Économistes de la
Santé Français, Décembre 2012, Reims, France. MARCOCCIA M. , « Parler politique dans un forum de discussion ", in Langage et société, $\mathrm{n}^{\circ} 104,2003$, pp. 9-55.

MARTIN T., QUIVIGER P-Y., Action médicale et confiance, Presses universitaires de FrancheComté, 2007.

MÉADEL C. , AKRICH M. , «Internet, tiers nébuleux de la relation patient-médecin », in Les Tribunes de la santé : Sève, Presses de Science Po, 2010, pp. 41-48.

MÉNARD J. , « L'autorité médicale », in De l'autorité, Colloque annuel 2007, Antoine Compagnon, Odile Jacob, Paris, 2008.

MONTE M. , OGER C. (dir.), « Discours d'autorité : des discours sans éclat(s) ? », in Mots. Les langages du politique, $\mathrm{n}^{\circ} 107,2015$.

MORIARTY C.M. , STRYKER J.E. , « Prevention and screening efficacy messages in newspaper accounts of cancer ", in Health Education Research, Vol. 23, n³, 2008.

ORIGGI G. , « Croyance, déférence et témoignage », in La philosophie cognitive, E. Pacherie, J. Proust éd., Paris, 2004.

ORIGGI G., Qu'est ce que la confiance?, Paris, Vrin, 2008.

PINTE J-P. , Enseignement, préservation et diffusion des identités numériques, Hermès Lavoisier, 2014.

ROMEYER H. , «TIC et santé : entre information médicale et information de santé», in Tic\&société [En ligne], Vol. 2, $\mathrm{n}^{\circ} 1 \mid 2008$, mis en ligne le 13 octobre 2008, consulté le 8 juillet 2015. URL : http://ticetsociete.revues.org/365.

WEBER M., Économie etsociété, Paris, Plon, 1971. WILSON P., Second-hand knowledge. An inquiry into cognitive authority, Greenwood Press, 1983. 


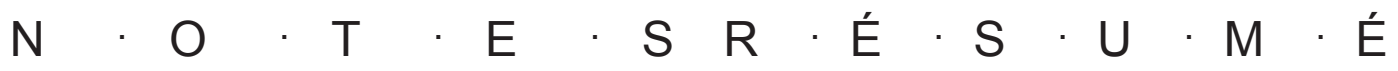

1. Hélène Romeyer (2008) distingue ainsi l'information médicale « traditionnellement produite par des spécialistes et répondant aux critères et au fonctionnement de l'information publique scientifique » et l'information de santé « à destination du grand public, diffusée sur Internet [qui] voit sa production échapper aussi bien au secteur médical qu'à l'État ».

2. http://www.lesimpatientes.com/forum. asp?cid $=15 \& \mathrm{id}=60$ \&pagecur $=0$

3. http://www.lesimpatientes.com/forum. asp? $\mathrm{cid}=14 \& \mathrm{id}=8$ \&pagecur $=0$

4. http://www.lesimpatientes.com/

5. Esquivel et al. (2006) s'intéressent à une liste de discussion non modérée consacrée au cancer de sein : sur 4600 messages postés en quatre mois, seuls dix messages contenaient des informations fausses, lesquelles ont été corrigées dans un délai allant d'une demi-heure à quatre heures.
Dans le domaine de la santé, l'autorité médicale est incarnée par le médecin, véritable "figure d'autorité » (Herfray, 2005) par son statut professionnel et la légitimité sociale dont il bénéficie. Pourtant, de nombreux facteurs tendent à remettre en cause cette autorité, parmi lesquels l'apparition de technologies permettant aux patients de s'informer « autrement » et favorisant une mise à distance de l'information médicale. Dans cet article nous avons choisi de travailler à partir de deux forums de discussion publiés sur le site « Les Impatientes » soumis à une analyse de discours permettant de mettre en évidence les procédés discursifs de remise en cause de l'autorité médicale et les postures de légitimité adoptées par les patientes-internautes. Notre objectif est ainsi de mieux comprendre comment se construit, à travers les discours et les dispositifs d'énonciation, une autorité d'expérience au sein d'une communauté en ligne où l'information de santé vient, non pas suppléer, mais expliciter, éclairer et compléter l'information médicale.

\begin{abstract}
In the health field, medical authority is personified by the medical doctor, a genuine "authority figure" (Herfray, 2005) by his professional status and the social legitimacy he enjoys. However, many factors tend to relativize this authority, among them the emergence of technologies that allow patients to access medical information beside medical doctors, and thus tend to favor distanciation and critical postures. In this article we chose to work on two forums published on the website «Les impatientes». Using discourse analysis, we attempt to highlight the discursive processes that question medical authority and the legitimacy postures adopted by patients-Internet users. Our aim is to understand how is constructed, through discourses
\end{abstract}


and enunciation devices, an experience authority in an online community where health information doesn't substitute but explains, clarifies and completes medical information. 